\title{
Patterns of Microvascular Response to Refractory Shock and Their Modulation by Vasoactive Resuscitations
}

El Rasheid Zakaria ${ }^{1 *}$, Bellal Joseph ${ }^{1}$, Faisal S Jehan ${ }^{1}$, Muhammad Khan ${ }^{1}$, Abdelrahman Algamal ${ }^{2}$, Faheem Sartaj ${ }^{2}$, Muhammad Jaffar Khan ${ }^{3}$ and Rajvir Singh ${ }^{3}$ ${ }^{1}$ Division of Trauma, Critical Care, Burns \& Emergency Surgery, The University of Arizona, Tucson, AZ 85721, USA

${ }^{2}$ Biomedical Research Center \& College of Arts and Health Sciences, Qatar University, P.O. Box 2713, Doha, Qatar

${ }^{3}$ Hamad Medical Corporation, P.O. Box 3050, Doha, Qatar

*Corresponding author: El Rasheid Zakaria, Division of Trauma, Critical Care, Burns \& Emergency Surgery, The University of Arizona, Tucson, AZ 85721, USA, Tel: +520 626-9010; Fax: +520 626 5016; E-mail: drelzak@surgery.arizona.edu

Received Date: December 10, 2017; Accepted Date: January 16, 2018; Published Date: January 20, 2018

Copyright: (C2018 Zakaria ER, et al. This is an open-access article distributed under the terms of the Creative Commons Attribution License, which permits unrestricted use, distribution, and reproduction in any medium, provided the original author and source are credited.

\begin{abstract}
Objective: Progressive hemorrhagic shock (HS) causes splanchnic vasoconstriction and hypo-perfusion together with profound depletions of cellular cytosolic energy phosphates (ATP). Cellular energy failure and the splanchnic hypo-perfusion are critical to the pathogenesis of shock decompensation and the subsequent death from cardiocirculatory arrest. We recently demonstrated post-resuscitation survival benefit for cellular cytosolic ATP replenishment but not for vasopressors use in a rat model of refractory HS. The aim of this study was to determine the effects of progressive HS on the splanchnic microvasculature, and to compare the effects of adjuvant resuscitations with either norepinephrine, vasopressin or lipid vesicles encapsulating ATP (ATPv) on this microvasculature.
\end{abstract}

Methods: 40-male Sprague-Dawley rats were randomized into 4 groups of 10 each: HS/conventionalresuscitation (CR), HS/CR+Norepinephrine, HS/CR+Vasopressin, and HS/CR+ATPv. HS=initial removal of $30 \%$ of the calculated blood volume plus subsequent transection of the spleen for uncontrolled haemorrhage until a predefined intervention set-point was achieved; $C R=$ shed blood returned+double the shed blood volume as lactated Ringer's solution. Four-level intestinal microvascular A1 through A4 arterioles (100-8 $\mu \mathrm{m}$ diameter) in the terminalileum were continuously monitored with intravital-microscopy, and their diameters were measured at baseline, during shock, after completion of resuscitation, and during a $2 \mathrm{~h}$ post-resuscitation observation period. In addition, we recorded measurements of the mean arterial pressure, shock index, blood-gas profiles, and complete metabolic panels.

Results: Progressive HS caused bimodal arteriole responses with progressive vasoconstriction from baseline of first-order A1 and second-order A2 $(-22.1 \pm 1.9 \%)$, a progressive vasodilation of the third-order A3 and fourth-order A4 arterioles $(+22.2 \pm 2.8 \%)$. Resuscitation initially restored A1 and A2 diameters to near baseline. This was followed by post-resuscitation $A 1$ and $A 2$ vasoconstriction in all groups, except the ATPv group $(-8.1 \pm 3.4 \%)$. The hemorrhage-induced vasodilation of the $\mathrm{A} 3$ and $\mathrm{A} 4$ arterioles was maintained during the post-resuscitation observation period in the CR $(+55.3 \pm 6.4 \%)$ and the ATPv groups $(+39.5 \pm 5.2 \%)$, but remarkably attenuated in the norepinephrine $(+9.6 \pm 5.8 \%)$ and vasopressin $(+9.4 \pm 8.8)$ groups.

Conclusions: Severe hemorrhagic shock causes specific splanchnic microvascular bimodal response that is dependent on arteriolar level. First-order A1 and second-order A2 arterioles exhibit progressive vasoconstriction, whereas, third-order A3 and fourth-order A4 arterioles show progressive vasodilation. Temporary administration of either norepinephrine or vasopressin along with conventional resuscitation exerted deleterious effects at all splanchnic microvascular levels by virtue of their peripheral vascular actions. The cellular cytosolic ATP replenishment maintained a better microvascular profile when compared with the two vasopressors.

Keywords: Progressive haemorrhagic shock; Cellular resuscitation; Intestinal microcirculation; Norepinephrine; Vasopressin; ATP

\section{Introduction}

The Committee on Trauma of the American College of Surgeons defined class-IV of a haemorrhagic shock as loss of $40 \%$ or more of the circulating blood volume [1]. Depending on the rate of blood loss, this class of shock can rapidly progress to a refractory shock that will not respond to resuscitation efforts leading to death from cardiocirculatory arrest. The pathogenesis of the cardiovascular collapse that is associated with a refractory shock stems from the inability of the heart and vasculature, respectively, to maintain contractility and vascular tone in order to sustain an effective blood pressure. Both heart contractility and vascular control mechanisms are energy-dependent physiologic functions that cannot be maintained or sustained during haemorrhagic shock (HS) due to the lack of cellular energy. It is well established that HS causes profound depletions of cellular cytosolic adenosine nucleotides $[2,3]$. As the cellular energy levels fall below the cellular metabolic demands, all energy-dependent cellular processes cease to operate leading to ionic disequilibrium, paradoxical cellular swelling, cellular dysfunction, and lactic acidosis [4,5]. Therefore, 
depletion of cellular adenosine 5'-triphosphate (ATP) and a persistent hypotension are central to the pathogenesis of the cardio-circulatory collapse that is associated with a refractory haemorrhagic shock. In addition, HS also causes splanchnic vasoconstriction and hypoperfusion [6,7]. While methods of resuscitation including massive transfusion protocols can potentially correct hypotension, there is no prospective or experimental evidence to support recovery of the cellular ATP levels and restoration of the splanchnic perfusion with conventional resuscitation protocols.

It is not uncommon that in a progressive or refractory HS, vasopressors are temporarily used together with the definitive resuscitation protocol to manage a persistent hypotension that is not corrected by the aggressive resuscitation effort, and to prevent an imminent cardio-circulatory arrest [8]. However, the use of vasopressors in hypovolemic shock is controversial because their microvascular vasoconstriction action may worsen a pre-existing haemorrhage-induced splanchnic tissue hypo-perfusion [9]. By virtue of their peripheral vascular action, vasopressors increase the blood pressure at the cost of remarkable splanchnic vasoconstriction. In contrast to vasopressors use, the exogenous administration of either ATP or creatine phosphate seems to exert tissue protective effects during ischemia in various models and species [10-13]. However, the systemic administration of exogenous energy molecules during the resuscitation of HS is limited by two facts: 1) there is no experimental evidence to support intercellular ATP uptake by diffusion or by carriermediated transport; and 2) the half-life of ATP in the blood is less than 40 seconds due to the ectonucleotidase activity, which limits the biologic activity of ATP as an energy substrate [14]. These limitations were resolved in recent years by the development of ATPv, which is a product based on the concept of nanotechnology. ATPv are specially formulated, highly fusogenic, unilamellar lipid vesicles encapsulating ATP. Through the contact and subsequent rapid fusion of the lipid vesicles with cell membrane, the vesicles deliver their ATP directly into the cytosol of cell. In a rat model of traumatic haemorrhage, we established that the adjuvant administration of $\mathrm{ATPv}$ in underresuscitated animals restored and maintained hemodynamics by a mechanism related to a strong positive inotropic action of ATP [15].

Systematic studies that directly compare the peripheral microvascular action of vasopressors and ATPv as adjuvant resuscitations of progressive haemorrhagic shock are lacking. Therefore, we were prompted to utilize our direct intravital microscopy technique to determine, in real time, the splanchnic microvascular response profiles of norepinephrine, vasopressin, and ATPv as adjuvant resuscitations for progressive haemorrhagic shock. We hypothesized that progressive haemorrhagic shock has deleterious effects on the splanchnic microvascular functions, which are not corrected by conventional resuscitation or by norepinephrine or vasopressin as adjuvants to conventional resuscitation. We further hypothesized that the addition of ATPv to conventional resuscitation as a technique to replenishing endothelial and endocardial cytosolic energy will have better splanchnic microvascular profiles as compared with vasopressors.

\section{Materials and Methods}

\section{General animal preparation}

The Institutional Animal Care and Use Committees (IACUC) of Hamad Medical Corporation and Qatar University (Doha, Qatar) preapproved the experimental protocols. All animals received humane care in accordance with The Guide for the Care and Use of Laboratory Animals (National Research Council, 8th ed., 2011). Male SpragueDawley rats weighing $\sim 200 \mathrm{~g}$ were obtained from Harlan (Harlan laboratories, Correzzana, Italy). The animals were quarantined for 2 weeks in a temperature and light-controlled environment during which time they had free access to standard rat chow food and water ad libitum. On the day of the experiment, we anesthetized each animal with an intraperitoneal injection of $50 \mathrm{mg} / \mathrm{kg}$ of Pentobarbitone (Troy Laboratories Pty Ltd, Plumpton, Australia). A surgical plane of anesthesia was maintained during the course of the experiment with a supplemental subcutaneous injection of $25 \%$ of the induction dose at $60 \mathrm{~min}$ intervals. All animals received a subcutaneous injection of $2 \mathrm{ml}$ of pre-warmed normal saline to compensate for fluid loss during surgery and animal preparation. Body temperature was maintained at $37^{\circ} \pm 0.5^{\circ} \mathrm{C}$ with a rectal probe and a servo-controlled heating pad (TC-1000, Temperature Controller, CWE Incorporated, Ardmore PA, USA). We undertook surgery after the loss of the blink and withdrawal reflexes. Upon performing a tracheostomy, a Polyethylene PE-240 tubing of $1.68 \mathrm{~mm} \times 2.39 \mathrm{~mm}$ (Scientific Commodities Inc., Lake Havasu City, AZ, USA) was introduced into the trachea to ensure patency of the upper airway; and the animal was allowed to breathe room air spontaneously. We cannulated the left carotid artery and then the right femoral vein with PE-50 tubing of $0.58 \mathrm{~mm} \times 0.97 \mathrm{~mm}$ (Instech Laboratories, Inc., Plymouth Meeting PA, USA) for continuous blood pressure recording using a calibrated pressure transducer connected to a blood pressure analyzer (BPA-400, DigiMed, Louisville, KY, USA), as well as for blood withdrawal and resuscitation. A mid-line laparotomy and left abdominal flank incisions were performed to provide access to the terminal ileum and the spleen. Following the protocol, we undertook intravital microscopy through the ileum and transected the spleen to induce an uncontrolled haemorrhage. Hemodynamics were continuously monitored and digitally recorded at $5 \mathrm{~min}$ intervals during the course of the experiment. We calculated the cardio-dynamic indices [Mean arterial pressure (MAP); Shock index (SI)] from the hemodynamic parameters and they were also recorded at 5 min intervals.

\section{Intestinal preparation for intravital microscopy}

Our team performed a standard small intestine preparation designed for splanchnic microcirculatory studies. Briefly, following a midline laparotomy, the terminal ileum was exteriorized with an intact neurovascular supply. Using the cecum as a landmark, a short segment of the terminal ileum was selected for intravital microscopy. We placed two ligatures on the selected segment at a distance of $2 \mathrm{~cm}$ apart to exclude collateral circulation. Then, the segment was returned to the abdominal cavity. We positioned each animal on its side on a custom designed animal board. This board is equipped with a $60-\mathrm{ml}$ Plexiglas tissue bath and a built-in optical port within the tissue bath for transillumination, a gas port for tissue bath $\mathrm{pH}$ control, and a thermistor using a feedback-controlled heating coil to maintain the bath temperature at $37^{\circ} \mathrm{C}$. The segment was re-exteriorized into the tissue bath containing non-vasoactive modified Krebs' solution composed of $6.92 \mathrm{~g} / \mathrm{L}$ sodium chloride, $0.44 \mathrm{~g} / \mathrm{L}$ potassium chloride, $0.37 \mathrm{~g} / \mathrm{L}$ calcium chloride, and $2.1 \mathrm{~g} / \mathrm{L}$ sodium bicarbonate. While suspended within the tissue bath, we opened the segment with a cautery pen along the longitudinal border of its anti-mesenteric side; and, the lumen was flushed with warmed saline to remove luminal contents. To prepare the study segment for trans-illumination, the upper flap of the opened segment was spread over the optical port of the tissue bath. The edges of the flap were affixed to the optical port within the tissue bath 
with 4-6 sutures. As it is of the utmost importance to eliminate variables such as bath $\mathrm{pH}$ that affect vascular reactivity during the experiment, we used continuous bubbling of the tissue bath solution with a mixture of $\mathrm{N}_{2}$ and $\mathrm{CO}_{2}$ via the gas port to maintain a $\mathrm{pH}$ control at $7.4 \pm 0.05$ as well as a temperature coil (which regulates bath temperature) control at $37 \pm 0.5^{\circ} \mathrm{C}$. We then positioned the animal and the board on the stage of an upright trinocular microscope (Axio Varioscope, Ziess Germany) fitted with a high definition digital camera (Model HV-D30 Hitachi Kokusai, Tokyo, Japan) for direct in vivo microscopy. The real-time streamline video images of the micro-vessels were projected on a calibrated high resolution computer monitor and automatically saved to the computer hard drive for offline microvascular diameter measurements using digital calipers. To characterize the terminal ileum microcirculation, we used the microvascular anatomy and the nomenclature as described by Bohlen and Gore-Figure-1 [16].

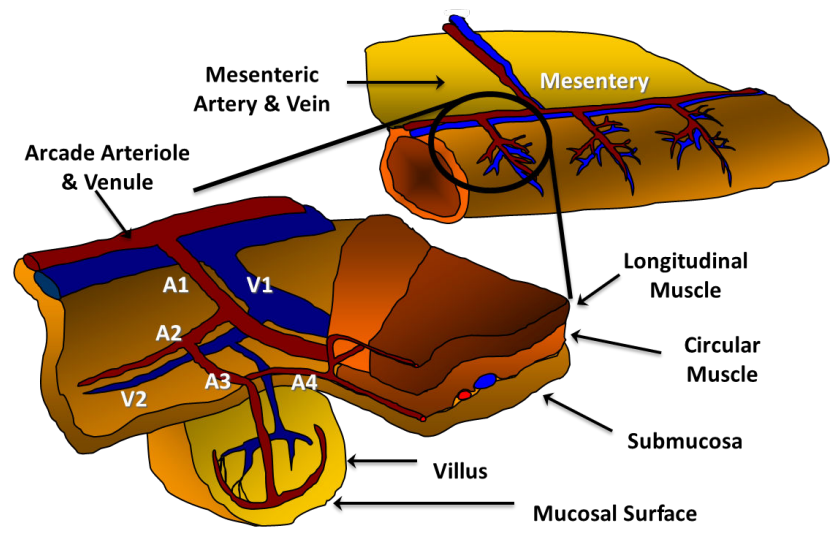

Figure 1: Anatomy and nomenclature of the terminal ileum microcirculation.

Briefly, this includes the first-order inflow arteriole (A1; $100 \mu \mathrm{m}$ diameter) branch from a mesenteric arcade arteriole acting as a feeding arteriole to the whole intestinal wall; two second-order arteriole (A2; $40-70 \mu \mathrm{m}$ diameter) branch from the $\mathrm{A} 1$ arteriole acting as transitional arterioles within the submucosal layer; and, the third-order arteriole (A3; 15-20 $\mu \mathrm{m}$ diameter) branch at a right angle from the A2 arteriole acting as a proximal pre-mucosal A3 arteriole and distal A4 precapillary arteriole $(8-12 \mu \mathrm{m}$ diameter) that terminates as a central villus arteriole. The criteria for an acceptable preparation of the intestine for intravital microscopy included a baseline MAP $>90$ $\mathrm{mmHg}$, at least three $\mathrm{A} 1$ arterioles in the trans-illuminated segment, a visually good $\mathrm{A} 1$ arteriole blood flow, and an active vasomotion in the arteriolar system. Animals that did not meet all these criteria during a 45 min equilibration period were scarified and not used in the study.

\section{Characterization of the model of class-IV hemorrhagic shock}

We performed our study in a reproducible rat model of traumatic exsanguination to simulate class-IV of haemorrhagic hypovolemic shock as defined by The Committee on Trauma of the American College of Surgeons [1]. This model encompasses significant tissue damage (a laparotomy and left-flank abdominal incisions plus transections of the splenic parenchyma and a vascular injury), and a reproducible shock. A class-IV haemorrhagic shock was induced with two successive phases of haemorrhage. Phase-1 involved pumpcontrolled venous withdrawal of $30 \%$ of the animal's calculated blood volume over 15 min using the formula of Lee and Blaufox [17]. A phase- 2 uncontrolled haemorrhage was induced by transections of the splenic parenchyma at the two ends of the organ as well as the severing of one of the branches of the splenic artery. The transected organ was returned to the abdominal cavity for free arterial and venous bleeding until a prior preset point for haemostasis and initiation of resuscitation intervention was reached. This was defined by a persistent mean arterial pressure $\leq 40 \mathrm{mmHg}$ and a persistent increase in the SI to a value $\geq 5$ for $10 \mathrm{~min}$ during the period of active uncontrolled abdominal bleeding. SI was calculated as the ratio of heart rate and systolic arterial pressure [18].

\section{Chemical, drugs, and tissue-bath solution}

All chemicals were purchased from the Sigma Chemical Company (St. Louis, Missouri, USA). The non-vasoactive Krebs solution used to continuously bath the intestinal segment during tissue preparation, the equilibration period, and during intravital microscopy contained 6.92 $\mathrm{g} / \mathrm{L}$ sodium chloride, $0.44 \mathrm{~g} / \mathrm{L}$ potassium chloride, $0.37 \mathrm{~g} / \mathrm{L}$ calcium chloride, and $2.1 \mathrm{~g} / \mathrm{L}$ sodium bicarbonate at a $\mathrm{pH}$ of 7.4 and osmolality of $285 \mathrm{mOsm} / \mathrm{L}$. We obtained Levophed (Norepinephrine Bitartrate, Hospira Inc., Lake Forest, IL, USA) and Pitressin (Synthetic Vasopressin, Par Pharmaceutical, Inc., Parsippany, NJ, USA), from Hamad Hospital Pharmacy. The energy delivery vehicle (ATPv) was purchased from Energy Delivery Solutions (Energy Delivery Solutions LLC, Jeffersonville IN USA). We added the designated vasopressor (or ATPv) to the crystalloid resuscitation fluid according to protocol. ATPv is a special formulation of Adenosine-5'-triphosphate (ATP) encapsulated in nanoscale delivery vehicles (lipid vesicles), which are highly fusogenic. In in vitro protocols, fusion of the lipid vesicles with the cell membrane and the rate of delivery of ATP directly into the cytosol of cells has been verified and validated by the manufacturer from the incubation of the vesicles with human umbilical endothelial cells, rat hepatocytes, and rat cardiomyocytes $[19,20]$. In these experiments, microscopic examination of fluorescent distribution within the human umbilical endothelial cells suggested that carboxyfluorescein delivery was diffuse rather than punctuated, indicating that vesicles simply were not aggregating on the cell surface but rather fusing with cell membranes. The amount of ATP that accumulated in the endothelial cells was dependent on the mole fraction of the fusogenic lipid of the vesicle, the concentration of ATPlipid vesicle used, the time of vesicle exposure, and the ATP concentration in the vesicles. Therefore, we assume that the effect of adjuvant resuscitation with ATPv in our work is attributed to direct intracellular ATP delivery as well as the free ATP in the ATPv formula.

\section{Experimental protocol and measurements}

In keeping with the experimental protocol depicted in Figure 2, initially forty animals were evenly divided between 4 resuscitation groups. Each underwent general surgical preparation, in addition to preparation of the terminal ileum for direct intravital microscopy. Following this, $45 \mathrm{~min}$ was allowed for systemic equilibration and recovery of the animal from surgical stress. During the equilibration period, hemodynamics and microvascular arteriolar diameters were continuously monitored and repeatedly measured to determine the 
baseline diameters for the four-level arterioles. A measurement of a baseline diameter was considered valid when the variability in the measurements within a $10 \mathrm{~min}$ interval was $<5 \%$. In addition to the baseline arteriolar diameters determination, the baseline measurements also included arterial blood chemistry; blood gas analysis, systolic, diastolic, and mean arterial pressures; heart rate; cardio-dynamic indices; rectal and bath temperatures; and, bath $\mathrm{pH}$. Following the baseline measurements, all animals underwent the first phase of a volume-controlled haemorrhage (Figure 2).

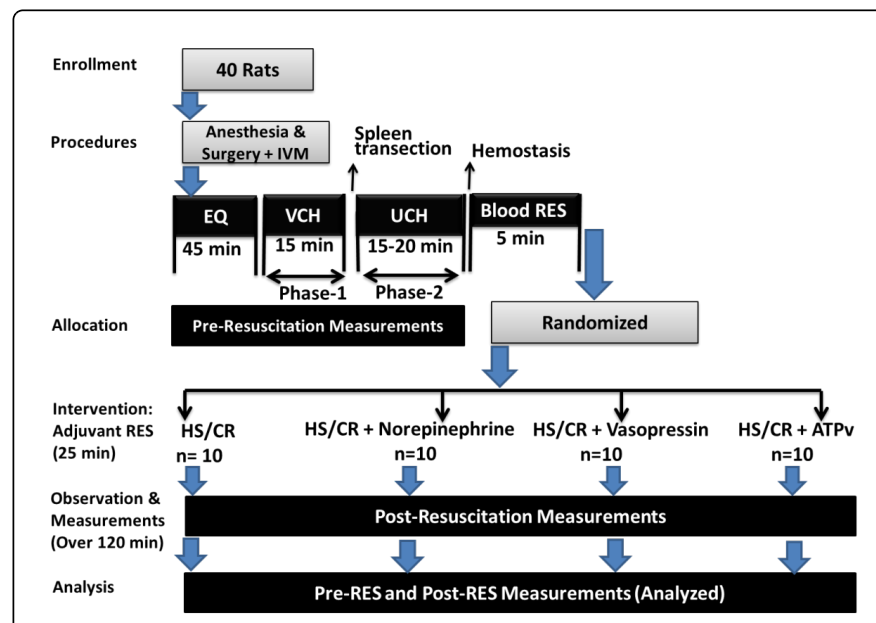

Figure 2: Timeline and experimental protocol.

Haemorrhage was initiated by a pump-controlled femoral vein blood withdrawal (Genie Touch, Kent Scientific Corporation, Torrington, CT, USA) totalling $30 \%$ of the calculated animal's blood volume over $15 \mathrm{~min}$. The shed blood was preserved in a heparincoated tube (Heparin Leo, 1000 I.U. per ml, Ballerup, Denmark) for later re-infusion, according to protocol. At the end of the first phase of haemorrhage, the second phase was immediately started by inducing spleen/vascular injuries as described earlier. During the activeuncontrolled haemorrhage, hemodynamics was closely monitored until the resuscitation intervention-trigger time point was reached, which was defined from a persistent MAP $\leq 40 \mathrm{mmHg}$ and a SI $\geq 5$ over a $10 \mathrm{~min}$ observation period during the active-uncontrolled haemorrhage (15-20 min). At this resuscitation intervention-trigger time point, haemostasis was rapidly achieved by ligation of the splenic pedicle. Following haemostasis, we initiated intravenous resuscitation by returning the shed blood over $5 \mathrm{~min}$. The animals were then randomly allocated to receive fluid resuscitation over the next $25 \mathrm{~min}$ with double the shed blood volume as Ringer's lactate [conventional resuscitation, CR] or with double the shed blood volume as ringer's lactate containing either norepinephrine $(25 \mu \mathrm{g} / 100 \mathrm{~g}$ body weight), vasopressin (0.8 units/100 g body weight), or, lipid vesicles encapsulating ATP, that is, ATPv ( $2.5 \mathrm{mM} / \mathrm{ml}$ lipid/ $2.5 \mathrm{mM}$ ATP $)$. The vasopressor dose as calculated based on the animal's body weight, was admixed with the ringer's lactate solution and infused over the $25 \mathrm{~min}$ following the complete return of the shed blood. One animal from the VP group died at the $2 \mathrm{~h}$ post-resuscitation time point. Hemodynamics and cardio-dynamic indices were continuously monitored and digitally recorded at $5 \mathrm{~min}$ intervals.

We assessed arterial blood chemistry at the baseline, at the end of the two haemorrhage phases, and at $2 \mathrm{~h}$ after completion of resuscitation (VetScan iSTAT 1, Abaxis, Union City, CA, USA). Intestinal microvascular diameters of the $\mathrm{A} 1, \mathrm{~A} 2, \mathrm{~A} 3$ and $\mathrm{A} 4$ arterioles were measured at the baseline; approximately $10 \mathrm{~min}$ intervals during the shock phase; $10 \mathrm{~min}$ after haemostasis and the return of shed blood; 20 min after completion of resuscitation; and at 20 min intervals during the $120 \mathrm{~min}$ post-resuscitation observation period. At the conclusion of the experiment, the animal was euthanized with an anesthetic overdose and potassium chloride solution, and tissues were harvested from the anterior abdominal muscle, ileum, liver, kidney, lung and heart. The tissues were dried at $60^{\circ} \mathrm{C}$ to constant weight in order to assess the total tissue water content.

\section{Data reduction and statistics}

Interval data was summarized in the form of mean and standard error of the mean (SEM). All data are presented as mean \pm SEM, unless otherwise stated. Microvascular diameter data were normalized and presented as a percentage change from the corresponding prehaemorrhage baseline diameter for each arteriole. We carried out a two-way ANOVA with repeated measurements of the experimental group, followed by Tukey's multiple comparisons test using GraphPad Prism version 6.07 for Windows (GraphPad Software, La Jolla California USA) in order to assess differences in the normalized arteriole diameter profile change between the groups and within the group. Likewise, we analyzed differences between the groups and within the group regarding MAP and SI, blood gas, and metabolic panel parameters at the baseline, after completion of the two phases of haemorrhage, and at the $2 \mathrm{~h}$ time point following haemostasis and resuscitation with a two-way ANOVA, followed by Tukey's multiple comparisons post-test (GraphPad Software, La Jolla California USA). Computed confidence intervals and statistical significance were corrected for multiple comparisons with Tukey's post hoc test.

\section{Results}

\section{Mean arterial pressure (MAP) and shock index (SI)}

Table- 1 depicts the MAP and the SI for the 4 resuscitation groups.

\begin{tabular}{|c|c|c|c|c|c|}
\hline $\begin{array}{l}\text { Cardio-dynamic } \\
\text { Index }\end{array}$ & HS/CR & $\begin{array}{l}\text { HS/CR+ } \\
\text { Norepinephrine }\end{array}$ & $\begin{array}{l}\text { HS/CR+ } \\
\text { Vasopressin }\end{array}$ & $\begin{array}{l}\text { HS/CR+ } \\
\text { ATPv }\end{array}$ & $\begin{array}{l}\text { P-Value } \\
\text { (Between Groups) }\end{array}$ \\
\hline \multicolumn{6}{|c|}{ Mean Arterial Pressure (MAP) } \\
\hline 0 & $127 \pm 7$ & $115 \pm 2$ & $114 \pm 3$ & $119 \pm 1$ & ns \\
\hline 1 & $39 \pm 5^{a}$ & $35 \pm 5^{a}$ & $35 \pm 6^{a}$ & $36 \pm 4^{a}$ & ns \\
\hline 2 & $100 \pm 21^{\mathrm{b}, \mathrm{d}}$ & $116 \pm 42^{\mathrm{d}}$ & $145 \pm 33^{\mathrm{b}, \mathrm{d}, \mathrm{g}, \mathrm{h}, \mathrm{i}}$ & $104 \pm 11^{d, h}$ & $\begin{array}{l}{ }^{g} p<0.0001 \text { vs. CR group } \\
h_{p}=0.0087 \text { vs. NE group }\end{array}$ \\
\hline
\end{tabular}


Citation: Zakaria El R, Joseph B, Jehan FS, Khan M, Algamal A, et al. (2018) Patterns of Microvascular Response to Refractory Shock and Their Modulation by Vasoactive Resuscitations. J Surg Anesth 2: 110.

Page 5 of 10

\begin{tabular}{|c|c|c|c|c|c|}
\hline & & & & & ${ }^{i} \mathrm{p}<0.0001$ vs. ATPv group \\
\hline 3 & $55 \pm 28^{\mathrm{c}, \mathrm{f}}$ & $75 \pm 25^{c, e, f}$ & $56 \pm 29^{c, e, f}$ & $84 \pm 22^{\mathrm{c}, \mathrm{e}, \mathrm{j}, \mathrm{k}}$ & $\begin{array}{l}\mathrm{j}=0.010 . v s \mathrm{CR} \\
\mathrm{k}=0093 v s . \mathrm{VP}\end{array}$ \\
\hline $\begin{array}{l}\text { P-Values } \\
\text { (within group) }\end{array}$ & $\begin{array}{l}a<0.0001 \\
b=0.043 \\
c<0.0001 \\
d<0.0001 \\
e=0.426 \\
f<0.0001\end{array}$ & $\begin{array}{l}a<0.0001 \\
b=0.99 \\
c=0.0001 \\
d<0.0001 \\
e<0.0001 \\
f<0.0001\end{array}$ & $\begin{array}{l}a<0.0001 \\
b=0.0034 \\
c<0.0001 \\
d<0.0001 \\
e<0.107 \\
f<0.0001\end{array}$ & $\begin{array}{l}a<0.0001 \\
b=0.335 \\
c=0.0010 \\
d<0.0001 \\
e<0.0001 \\
f=0.133\end{array}$ & \\
\hline \multicolumn{6}{|l|}{ Shock Index (SI) } \\
\hline 0 & $2.44 \pm 0.11$ & $2.45 \pm 0.14$ & $2.49 \pm 0.16$ & $2.42 \pm 0.17$ & ns \\
\hline 1 & $5.2 \pm 1.7^{\mathrm{a}}$ & $5.8 \pm 0.6^{a}$ & $5.3 \pm 0.8^{a}$ & $5.8 \pm 0.9^{a}$ & ns \\
\hline 2 & $2.8 \pm 0.7^{d}$ & $2.99 \pm 0.2^{\mathrm{d}}$ & $1.8 \pm 0.1^{\mathrm{d}, \mathrm{h}}$ & $2.9 \pm 0.1^{\mathrm{d}, \mathrm{k}}$ & $\begin{array}{l}{ }^{h} p=0.0128 \text { vs. NE group } \\
{ }^{k} p=0.0252 \text { vs. VP group }\end{array}$ \\
\hline 3 & $4.5 \pm 2.8^{\mathrm{c}, \mathrm{f}}$ & $4.0 \pm 0.3^{\mathrm{c}, \mathrm{e}, \mathrm{f}}$ & $5.0 \pm 0.7^{c, f}$ & $3.4 \pm 0.2^{\mathrm{c}, \mathrm{e}, \mathrm{j}, \mathrm{k}}$ & $\begin{array}{l}\mathrm{j}_{\mathrm{p}}=0.0393 \text { vs. CR group } \\
\mathrm{k}_{\mathrm{p}}=0.0003 \text { vs. VP group }\end{array}$ \\
\hline P-Value (within group) & $\begin{array}{l}a<0.0001 \\
b=0.99 \\
c<0.0001 \\
d<0.0001 \\
e=0.3678 \\
f<0.0001\end{array}$ & $\begin{array}{l}a<0.0001 \\
b=0.5034 \\
c=0.0006 \\
d<0.0001 \\
e<0.3678 \\
f=0.0473\end{array}$ & $\begin{array}{l}a<0.0001 \\
b=0.2713 \\
c<0.0001 \\
d<0.0001 \\
e=0.8137 \\
f<0.0001\end{array}$ & $\begin{array}{l}a<0.0001 \\
b=0.4824 \\
c=0.0357 \\
d<0.0001 \\
e<0.0001 \\
f=0.5654\end{array}$ & \\
\hline
\end{tabular}

Table 1: Variation trends in MAP and SI within the group and between groups; Cardio-dynamic indices (MAP and SI) presented as mean \pm SD: HS=Haemorrhagic Shock; CR=Conventional Resuscitation; 0, 1, 2, and 3 are values measured at baseline, after completion of the haemorrhage phases, at $10 \mathrm{~min}$ after completion of resuscitation; and at $2 \mathrm{~h}$ post-resuscitation, respectively. Variation trends within the group's time points and between groups were analyzed by two-way ANOVA and Tukey's post hoc test to correct for multiple comparisons. Comparisons within the group: 1 vs. 0 (a); 2 vs. 0 (b); 3 vs. 0 (c); 2 vs. 1 (d); 3 vs. 1 (e); and 3 vs. 2 (f). Comparisons between the groups: (g,h,i,j,k): CR=HS/CR group, NE=HS/CR +Norepinephrine group; VP=HS/CR+Vasopressin group; and ATPv=HS/CR+ATPv group.

There were no significant differences between groups in the MAP and SI at baseline and after completion of the two-phase haemorrhage $(\mathrm{P}>0.488)$. At the $10 \mathrm{~min}$ time-point following completion of resuscitation, the two vasopressor groups maintained significantly higher MAP $(\mathrm{P}<0.0001)$ than the conventional resuscitation and the adjuvant $\mathrm{ATPv}$ groups, whereas, the vasopressin group maintained a significantly lower SI $(\mathrm{P}=0.025)$ than the norepinephrine and the ATPv adjuvant resuscitation groups. At the $2 \mathrm{~h}$ time-point following haemostasis and resuscitation, only the norepinephrine and the ATPv adjuvant resuscitation groups maintained significantly higher MAP $(\mathrm{P}<0.01)$ than the conventional and the adjuvant vasopressin groups. However, only the adjuvant $\mathrm{ATPv}$ resuscitation group maintained a significantly lower SI $(\mathrm{P}=0.039)$ when compared with the conventional and the adjuvant vasopressin resuscitation groups. Within groups, MAP and SI differed much in accordance with haemorrhagic shock, resuscitation type and post-resuscitation observation time. Isolated vital signs are generally perceived as unreliable in the characterization of the level of shock due to the impact of the physiologic compensatory adjustments that are associated with blood loss. Instead, a composite of MAP and the Shock Index (SI) together with other metabolic indices has been advocated to better characterize the class of shock and to riskstratify resuscitation requirements. To assess the impact of this concept on our model, MAP and SI profiles during the entire experimental protocol for the four resuscitation groups were plotted and depicted in figure-3.
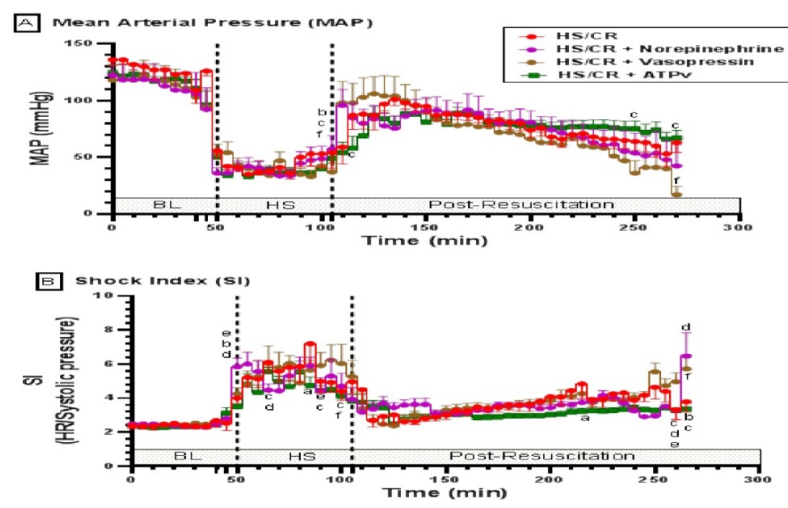

Figure 3: Mean Arterial Pressure (MAP), and Shock Index (SI). 
Citation: Zakaria El R, Joseph B, Jehan FS, Khan M, Algamal A, et al. (2018) Patterns of Microvascular Response to Refractory Shock and Their Modulation by Vasoactive Resuscitations. J Surg Anesth 2: 110.

Page 6 of 10

As shown in the figure, only the cytosolic energy replenishment group and the norepinephrine groups maintained better MAP and SI towards the end of the two hours post-resuscitation observation period.

\begin{tabular}{|c|c|c|c|c|c|}
\hline Metabolic Panel & HS/CR & $\begin{array}{l}\text { HS/CR+ } \\
\text { Norepinephrine }\end{array}$ & $\begin{array}{l}\text { HS/CR+ } \\
\text { Vasopressin }\end{array}$ & $\begin{array}{l}\text { HS/CR+ } \\
\text { ATPV }\end{array}$ & $\begin{array}{l}\text { P-value } \\
\text { (between groups) }\end{array}$ \\
\hline \multicolumn{6}{|l|}{ Sodium, $\mathrm{Na}$} \\
\hline 1 & $141.63 \pm 1.10$ & $140.30 \pm 0.78$ & $140.60 \pm 0.95$ & $140.70 \pm 0.40$ & ns \\
\hline 2 & $141.40 \pm 1.67$ & $137.30 \pm 1.06$ & $136.00 \pm 1.70^{a}$ & $134.00 \pm 2.48^{\mathrm{a}, \mathrm{d}}$ & 0.0033 \\
\hline 3 & $139.88 \pm 0.81$ & $140.30 \pm 1.24$ & $136.80 \pm 2.12$ & $138.30 \pm 1.35$ & ns \\
\hline P-value (within group) & ns & ns & 0.034 & 0.0012 & \\
\hline \multicolumn{6}{|l|}{ Potassium, $\mathrm{K}$} \\
\hline 1 & $4.10 \pm 0.16$ & $4.01 \pm 0.15$ & $4.22 \pm 0.23$ & $4.52 \pm 0.18$ & ns \\
\hline 2 & $6.45 \pm 0.52^{\mathrm{a}}$ & $6.09 \pm 0.38^{a}$ & $6.13 \pm 0.37^{a}$ & $6.99 \pm 0.40^{a}$ & ns \\
\hline 3 & $5.35 \pm 0.25^{b, c}$ & $4.97 \pm 0.26^{b, c}$ & $5.40 \pm 0.43^{b}$ & $5.09 \pm 0.33^{b}$ & ns \\
\hline P-value & $<0.028$ & 0.032 & 0.007 & $<0.0001$ & \\
\hline \multicolumn{6}{|l|}{ Chloride, $\mathrm{Cl}$} \\
\hline 1 & $108.13 \pm 1.39$ & $104.80 \pm 0.80$ & $104.40 \pm 1.29$ & $104.70 \pm 0.70$ & ns \\
\hline 2 & $116.75 \pm 1.22^{a}$ & $113.60 \pm 2.73^{a}$ & $111.20 \pm 2.31$ & $109.40 \pm 2.42^{f}$ & 0.015 \\
\hline 3 & $112.38 \pm 2.01$ & $112.40 \pm 3.30^{b}$ & $109.60 \pm 2.84$ & $109.70 \pm 1.22$ & ns \\
\hline P-value (within group) & 0.002 & $<0.039$ & ns & ns & \\
\hline \multicolumn{6}{|l|}{ Anion Gap } \\
\hline 1 & $12.50 \pm 0.82$ & $14.80 \pm 0.58$ & $15.40 \pm 0.51$ & $15.50 \pm 0.76$ & ns \\
\hline 2 & $22.32 \pm 0.99^{a}$ & $23.40 \pm 1.44^{a}$ & $20.00 \pm 3.22^{a}$ & $25.22 \pm 0.80^{a}$ & ns \\
\hline 3 & $17.00 \pm 1.82^{b, c}$ & $17.60 \pm 1.29^{c}$ & $16.00 \pm 3.48^{c}$ & $18.80 \pm 1.16^{c}$ & ns \\
\hline P-value & $<0.017$ & $<0.015$ & $<0.015$ & $<0.0003$ & \\
\hline \multicolumn{6}{|c|}{ Blood Urea Nitrogen, BUN } \\
\hline 1 & $13.50 \pm 1.34$ & $17.40 \pm 1.97$ & $13.00 \pm 1.52$ & $16.30 \pm 1.79$ & ns \\
\hline 2 & $17.00 \pm 1.17^{\mathrm{a}}$ & $19.00 \pm 3.21^{a}$ & $13.60 \pm 1.83^{a}$ & $17.15 \pm 1.97^{a}$ & ns \\
\hline 3 & $19.00 \pm 2.29^{b, c}$ & $20.00 \pm 1.64^{c}$ & $17.18 \pm 4.86^{c}$ & $17.70 \pm 1.25^{c}$ & ns \\
\hline P-value (within group) & $<0.017$ & $<0.015$ & $<0.015$ & $<0.0003$ & \\
\hline \multicolumn{6}{|l|}{ Hemoglobin } \\
\hline 1 & $14.26 \pm 0.83$ & $14.49 \pm 0.68$ & $14.71 \pm 0.29$ & $14.95 \pm 0.26$ & ns \\
\hline 2 & $8.34 \pm 1.15^{a}$ & $7.46 \pm 0.84^{a}$ & $7.42 \pm 0.57^{\mathrm{a}}$ & $6.04 \pm 0.40^{\mathrm{a}}$ & ns \\
\hline 3 & $13.65 \pm 0.64^{c}$ & $12.72 \pm 0.40^{\mathrm{b}, \mathrm{c}}$ & $12.54 \pm 0.81^{\mathrm{b}, \mathrm{c}}$ & $11.30 \pm 0.41^{\mathrm{b}, \mathrm{c}}$ & ns \\
\hline P-value (within group) & $<0.0001$ & $<0.023$ & $<0.0041$ & $<0.0001$ & \\
\hline \multicolumn{6}{|l|}{ Hematocrit } \\
\hline 1 & $42.00 \pm 2.44$ & $48.8 \pm 5.53$ & $48.30 \pm 5.36$ & $44.00 \pm 0.76$ & ns \\
\hline
\end{tabular}

\section{Metabolic panel and arterial blood gas}

Table-2 and Table-3 show the complete metabolic panel and the arterial blood gas analyses. 
Citation: Zakaria El R, Joseph B, Jehan FS, Khan M, Algamal A, et al. (2018) Patterns of Microvascular Response to Refractory Shock and Their Modulation by Vasoactive Resuscitations. J Surg Anesth 2: 110.

Page 7 of 10

\begin{tabular}{|l|l|l|l|l|l|}
\hline 2 & $24.50 \pm 3.37^{\mathrm{a}}$ & $30.40 \pm 7.84^{\mathrm{a}}$ & $21.80 \pm 1.67^{\mathrm{a}}$ & $17.80 \pm 1.18^{\mathrm{a}}$ & $\mathrm{ns}$ \\
\hline 3 & $40.13 \pm 1.89^{\mathrm{c}}$ & $43.70 \pm 6.03 \mathrm{c}$ & $36.80 \pm 2.34^{\mathrm{b}, \mathrm{c}}$ & $33.20 \pm 1.20^{\mathrm{b}}$ & $\mathrm{ns}$ \\
\hline P-value (within group) & $<0.0003$ & $<0.0006$ & $<0.0033$ & $<0.0060$ & \\
\hline
\end{tabular}

Table 2: Metabolic profile trends within the group and between the groups; Data are presented as mean \pm SEM: HS=Haemorrhagic Shock; $\mathrm{CR}=$ Conventional Resuscitation; 1,2, and 3 values measured at the pre-haemorrhage baseline, after completion of the haemorrhage phases, and at $2 \mathrm{~h}$ post-resuscitation, respectively. Variation trends within the group's time points and between groups were measured by two-way ANOVA and Tukey's post hoc test to correct for multiple comparisons. Comparisons within the group: 2 vs. 1 (a); 3 vs. 1 (b); and for 3 vs. 2 (c). Comparisons between groups: (d) $\mathrm{P}=0.0033$ vs. the HS/CR group, and (f) $\mathrm{P}=0.015$ vs. the HS/CR group.

\begin{tabular}{|c|c|c|c|c|c|}
\hline Arterial Blood Gas & HS/CR & $\begin{array}{l}\text { HS/CR+ } \\
\text { Norepinephrine }\end{array}$ & $\begin{array}{l}\text { HS/CR+ } \\
\text { Vasopressin }\end{array}$ & $\begin{array}{l}\text { HS/CR+ } \\
\text { ATPv }\end{array}$ & P-value \\
\hline \multicolumn{6}{|l|}{$\mathrm{pH}$} \\
\hline 1 & $7.34 \pm 0.01$ & $7.36 \pm 0.02$ & $7.38 \pm 0.01$ & $7.36 \pm 0.01$ & ns \\
\hline 2 & $7.00 \pm 0.05^{a}$ & $7.10 \pm 0.04^{\mathrm{a}}$ & $7.08 \pm 0.05^{a}$ & $7.00 \pm 0.04^{\mathrm{a}}$ & ns \\
\hline 3 & $7.22 \pm 0.05^{b, c}$ & $7.29 \pm 0.03^{c}$ & $7.21 \pm 0.03^{b, c}$ & $7.29 \pm 0.03^{c}$ & ns \\
\hline P-value & $<0.035$ & $<0.0001$ & 0.008 & $<0.0001$ & \\
\hline \multicolumn{6}{|l|}{ PCO2 } \\
\hline 1 & $46 \pm 2.76$ & $41 \pm 4.65$ & $40 \pm 1.31$ & $44 \pm 1.80$ & ns \\
\hline 2 & $34 \pm 8.50^{\mathrm{a}}$ & $21 \pm 1.52^{\mathrm{a}}$ & $21 \pm 3.30^{a}$ & $25 \pm 2.91^{a}$ & ns \\
\hline 3 & $39 \pm 5.10$ & $33 \pm 1.91^{c}$ & $31 \pm 2.85^{b, c}$ & $29 \pm 2.65^{c}$ & ns \\
\hline P-value & 0.018 & 0.011 & 0.045 & 0.0009 & \\
\hline \multicolumn{6}{|l|}{$\mathrm{HCO} 3$} \\
\hline 1 & $25 \pm 1.47$ & $26 \pm 1.28$ & $24 \pm 0.69$ & $25 \pm 0.89$ & ns \\
\hline 2 & $8 \pm 1.77^{\mathrm{a}}$ & $7 \pm 0.54^{a}$ & $6 \pm 1.13^{\mathrm{a}}$ & $7 \pm 0.83^{a}$ & ns \\
\hline 3 & $13 \pm 1.99^{\mathrm{b}, \mathrm{c}}$ & $16 \pm 1.22^{\mathrm{b}, \mathrm{c}}$ & $13 \pm 2.23^{b, c}$ & $15 \pm 1.27^{b, c}$ & ns \\
\hline P-value & $<0.017$ & 0.0001 & 0.0002 & 0.0001 & \\
\hline \multicolumn{6}{|c|}{ TCO2 (total carbon dioxide) } \\
\hline 1 & $27 \pm 1.52$ & $27 \pm 0.80$ & $25 \pm 0.70$ & $26 \pm 0.96$ & ns \\
\hline 2 & $9 \pm 1.89^{a}$ & $8 \pm 0.56^{a}$ & $8 \pm 1.63^{a}$ & $7 \pm 0.90^{a}$ & ns \\
\hline 3 & $17 \pm 2.18^{\mathrm{b}, \mathrm{c}}$ & $18 \pm 1.52^{b, c}$ & $14 \pm 2.04^{b, c}$ & $16 \pm 1.33^{b, c}$ & ns \\
\hline P-value & $<0.0001$ & $<0.0001$ & $<0.0001$ & $<0.0001$ & \\
\hline \multicolumn{6}{|l|}{ Base Excess } \\
\hline 1 & $-0.63 \pm 1.43$ & $-0.30 \pm 0.72$ & $-1.20 \pm 0.68$ & $-0.600 \pm 0.90$ & ns \\
\hline 2 & $-23.25 \pm 1.98^{a}$ & $-18.69 \pm 2.87^{a}$ & $-22.78 \pm 1.54^{a}$ & $-24.70 \pm 1.31^{a}$ & ns \\
\hline 3 & $-12.00 \pm 2.38^{b, c}$ & $-8.30 \pm 1.21 b, c$ & $-14.10 \pm 2.69^{b, c}$ & $-11.70 \pm 1.53^{b, c}$ & ns \\
\hline P-value & $<0.0001$ & $<0.0002$ & $<0.0001$ & $<0.0001$ & \\
\hline
\end{tabular}

Table 3: Arterial blood gas profile trends within the group and between the groups; Data are presented as mean \pm SEM: HS=Haemorrhagic Shock; $\mathrm{CR}=$ Conventional Resuscitation; 1, 2, and 3 were values measured at the pre-haemorrhage baseline, after completion of the haemorrhage phases, 
Citation: Zakaria El R, Joseph B, Jehan FS, Khan M, Algamal A, et al. (2018) Patterns of Microvascular Response to Refractory Shock and Their Modulation by Vasoactive Resuscitations. J Surg Anesth 2: 110.

Page 8 of 10

and at $2 \mathrm{~h}$ post-resuscitation, respectively. Variation trends within the group's time points and between groups were measured by two-way ANOVA and Tukey's post hoc test to correct for multiple comparisons. Comparisons within the group: 2 vs. 1 (a); 3 vs. 1 (b); and for 3 vs. 2 (c).

There were no differences between the four groups in the prehaemorrhage baseline metabolic parameters, blood gas, and acid base status. In all four groups, haemorrhagic hypovolemic shock remarkably decreased haemoglobin and haematocrit, produced a metabolic acidosis characterized by a low $\mathrm{pH}, \mathrm{PCO}_{2}, \mathrm{HCO}_{3}, \mathrm{TCO}_{2}$, (total carbon dioxide) and resulted in an increase in the base deficit as compared with the baseline pre-haemorrhage levels. These measurements suggested a similar class of shock in all four groups. None of the four resuscitation methods restored parameters of the metabolic panel, blood gas, or $\mathrm{pH}$ to the pre-haemorrhage baseline levels.

\section{Intestinal microvascular response profiles}

Figure 4 and Figure 5 depict the intestinal microvascular response profile of the four-level arterioles during the experimental protocol.
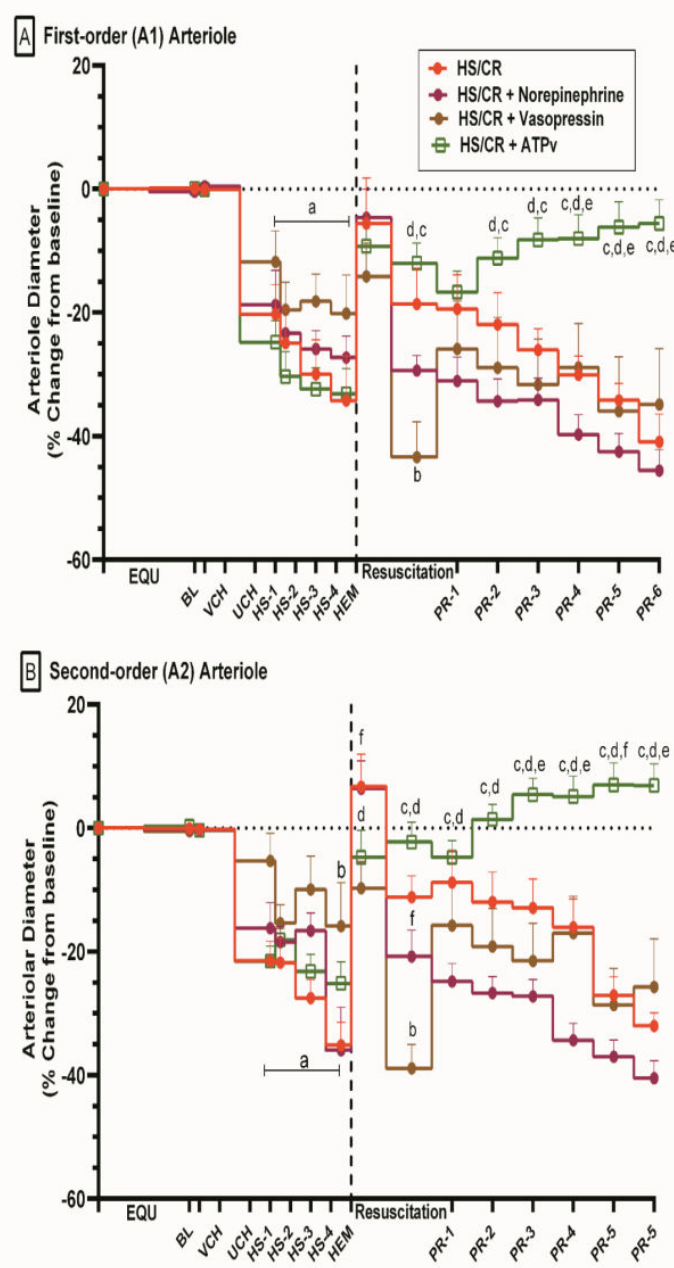

Figure 4: Intestinal inflow A1 and branching A2 arteriolar diameter changes during progressive hemorrhage and after resuscitation.

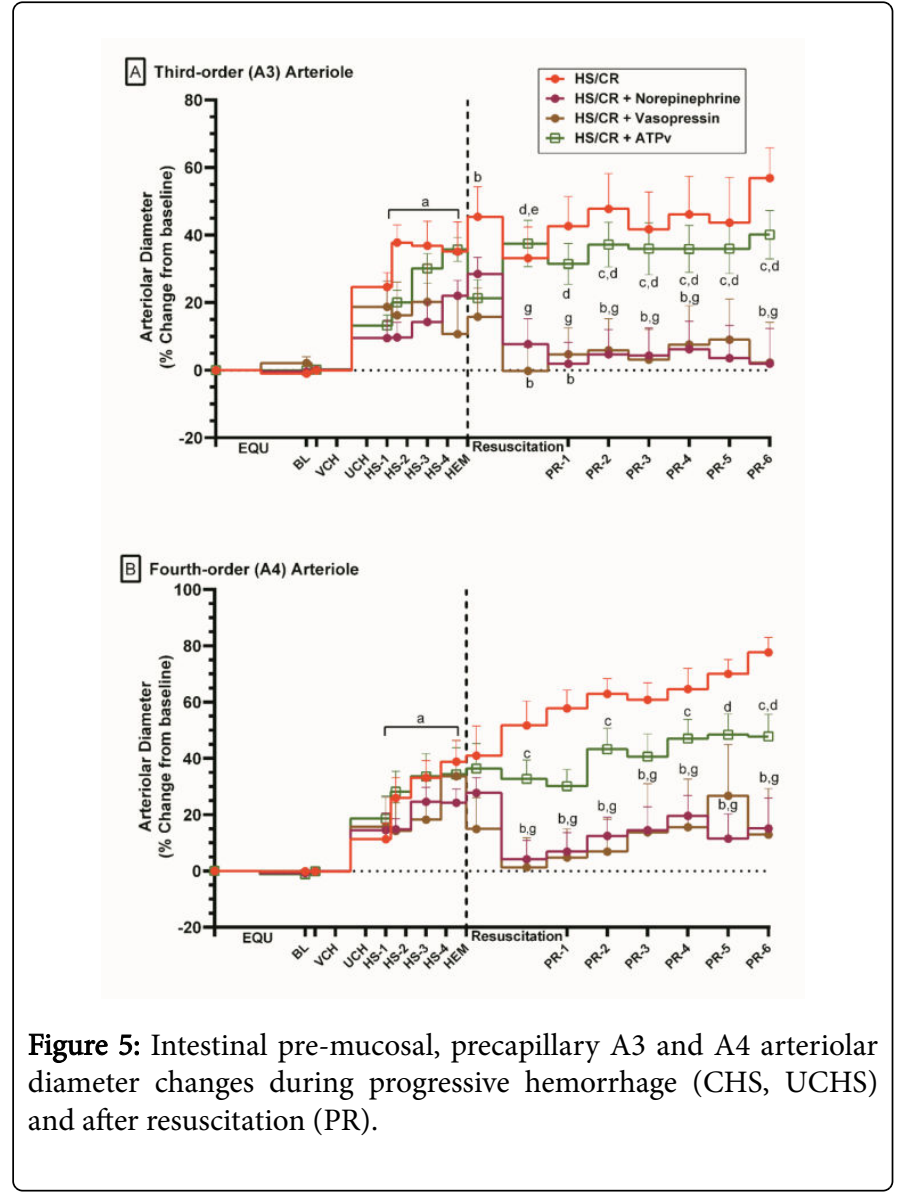

In all four groups, class-IV haemorrhagic hypovolemic shock induced bimodal intestinal microvascular responses characterized by remarkable vasoconstriction from the pre-haemorrhage baseline diameter of the larger inflow A1 and A2 arterioles (individual animal vs corresponding baseline arteriole diameter; $\mathrm{P}$ for the four groups $=\mathrm{P}<0.001$ ), and a remarkable vasodilation from the prehaemorrhage baseline diameter of the smaller pre-mucosal A3 and A4 arterioles (individual animal vs. corresponding baseline arteriole diameter; $\mathrm{P}$ for the four groups $=\mathrm{P}<0.001)$. Although blood flow in individual intestinal arterioles was not measured, distinct patterns of flow can be observed from the continuous real-time online video observation. We observed that blood flow in any of the 4 level arterioles does not follow the expected change in flow as predicted from the change in arteriolar diameter. In the pre-capillary A3 and A4 arterioles patterns of stop-flow-reflow, and reverse flow were frequently observed during the shock phase. These flow patterns were corrected during the post-resuscitation phase. However, both vasopressors maintained rather slow blood flow rates in the A3 and A4 arterioles during the post-resuscitation observation period. In all four groups, haemostasis and the return of shed blood restored diameters of the larger inflow A1 and A2 arterioles to near pre-haemorrhage baseline levels without affecting the haemorrhage-induced progressive vasodilation of the smaller pre-mucosal A3 and A4 arterioles (Figure 5). During the active resuscitation phase, vasopressin maintained a 
remarkable vasoconstriction of both the inflow A1 and branching A2 arterioles (HS/CR vs. HS/CR+Vasopressin group and HS/CR + Vasopressin vs. HS/CR+ATPv group; $\mathrm{P}$ for $\mathrm{A} 1$ arteriole $=\mathrm{P}<0.01)$. As a transitional arteriolar level, A2 even showed more variability during the active resuscitation phase (multiple comparisons between the four groups; $\mathrm{P}$ for the branching A2 arteriol $=\mathrm{P}<0.001$ ). During the postresuscitation observation period, the microvascular arteriolar response profile of the four groups differed according to the adjuvant resuscitation type (multiple comparisons between the four groups; $\mathrm{P}$ for the inflow $\mathrm{A} 1$ and the branching $\mathrm{A} 2$ arteriole response overtime $=\mathrm{P}<0.001$ ). Conventional resuscitation or adjuvant resuscitation with either norepinephrine or vasopressin produced progressive post-resuscitation vasoconstriction of the larger A1 and A2 arterioles, which was not seen in the ATPv resuscitation group (Figure 4). Whereas, the pressure-support resuscitation with either norepinephrine or vasopressin reversed the pre-existing haemorrhageinduced vasodilation of the smaller pre-mucosal A3 and A4 arterioles by inducing remarkable vasoconstriction of both arterioles during the entire post-resuscitation observation period (Figure 5). These postresuscitation pre-mucosal arteriolar response were not seen in the conventional or ATPv resuscitation groups.

\section{Discussion}

As previously demonstrated by intravital microscopy, moderate blood loss selectively constricts the first-order intestinal inflow arterioles and spares the smaller pre-mucosal precapillary arterioles. However, after resuscitation and correction of the volume deficit, progressive vasoconstriction at all intestinal arteriolar levels is typically observed [6,7,21-23]. In contrast, as the present study shows, progressive haemorrhagic hypovolemic shock, produces a bimodal pattern of intestinal microvascular reactivity that is neither altered nor reversed by traditional volume deficit correction methods. We presented direct experimental evidence supporting unfavourable microvascular effects of either norepinephrine or vasopressin on the pre-existing haemorrhage-induced bimodal intestinal microvascular response. We provided prove of concept that a precision resuscitation strategy presumably targeting the direct replenishments of the endothelial and endocardial cytosolic energy stores favourably alters this bimodal microvascular reactivity pattern.

Traumatic exsanguination leading to progressive hypotensive hypovolemic shock is a clinical crisis characterized by intensified cardinal signs of shock including profound hypotension, decreased cardiac output and a critical pulse. At the molecular, cellular, and tissue levels, a progressive hypotensive shock also manifests depletion of cellular energy stores, inflammation, lactic acidosis, a metabolic deficit, and inadequate end-organ perfusion [24]. Hypotension and central hemodynamics are largely amenable to correction by aggressive resuscitations including a temporary administration of vasopressors. In contrast, aggressive resuscitations often fail to recover or correct the sequels of exsanguination at the molecular, cellular and tissue levels. Therefore, the use of vasopressors for pressure-support resuscitation of hypovolemic shock, should always consider the unseen end-organ splanchnic microvascular vasoconstriction and hypo-perfusion that are associated with haemorrhagic shock. Markers of this microvascular dysfunction that largely determine resuscitation outcome are a persistent lactic acidosis, and a metabolic deficit. Two separate clinical studies have concluded that despite normalization of hemodynamics and urine output, tissue hypo-perfusion persisted in $80 \%$ to $85 \%$ of patients, as evidenced by lactic acidosis and decreased mixed venous oxygen saturation $[25,26]$. Other clinical studies have shown that the level and the rate of normalization of serum lactate (indirect measures of tissue oxygen utilization) correlated with mortality both in degree of elevation and in the time-dependent rate of normalization $[27,28]$. Based on the experimental and the clinical prospective evidences, the efficacy of resuscitation from any class of haemorrhagic shock should be redefined on the basis of restoration of end-organ perfusion rather than on the restoration of central hemodynamics. It should be emphasized that our present study is not a resuscitation outcome study, but designed to specifically determine the splanchnic microvascular reactivity during severe haemorrhagic shock, and to define the potential alteration of this reactivity during resuscitation. To fulfil this objective, all animals received the same class of shock and similar blood and crystalloid resuscitation volumes. Nevertheless, the specific bimodal arteriolar response during the haemorrhage phase, was altered differently during the resuscitation and the post-resuscitation observation phases, much in accordance with the peripheral microvascular action of the adjuvant resuscitation used.

Complete evaluation of the resuscitation outcome including survival was beyond the scope of the present study. Therefore, end-point resuscitation outcome measurements such as cardiac functions, markers and indices of the metabolic deficit and organ hypo perfusion were not simultaneously measured in this first prove of concept study. Similarly, the endothelial ATP turnover was not assessed in our study. Based on these limitations, no statement can be made about the prevailing endothelial and endocardial cytosolic energy balance during the $2 \mathrm{~h}$ post-resuscitation observation period. The ATPv used in our study was optimized to be highly fusogenic on contact with endothelial cells. In addition, the transport of ATP (Stokes-Einstein radius 3.48 $\mathrm{nm}$ ) across gap junctions is likely to be severely restricted based on the size selectivity of the para-cellular pathway of the capillary endothelial monolayer. Restriction to paracellular ATPv transport will even be greater. For these mechanistic reasonings, the ATPv will be totally excluded from the interstitial fluid volume. Thus, the favourable maintenance of the arterial pressure and the intestinal microvascular profile after ATPv can only be explained by the cellular cytosolic ATP replenishment.

By virtue of their vasoactive nature, both norepinephrine and vasopressin mediate their vascular effects by receptor-mediated mechanisms. Depending on the activation of their stimulated G protein-coupled receptors subtype, the resultant vasoactivity is determined by the receptor subtype being activated, its sensitivity, localization and function, and signal transduction mechanisms [29-31]. While the activation of the adrenergic receptors can promote both vasodilation as well as vasoconstriction, activation of the vasopressin receptors produces exclusive vasoconstriction. This is consistent with the unfavourable post-resuscitation microvascular profile of vasopressin as observed in our study. In contrast to adjuvant resuscitation with vasopressors, direct cytosolic energy replenishments with ATPv achieved the best post-resuscitation intestinal microvascular profile at a normal blood pressure. This favorable microvascular profile suggests that the haemorrhage-induced depletion of cellular energy stores, which is not recovered by either conventional or adjuvant vasopressors resuscitations, is a major mechanism of microvascular dysfunction and end-organ hypoperfusion [3,32]. ATPv exerts its microvascular effects by its encapsulated high energy phosphate (ATP) that is delivered directly into the cytosol of cells including the vascular endothelium and the endocardium. Adenosine, ATP, and ADP activate a family of purinoceptors subtypes for broader favourable functions ranging from 
regulation of myocardial $\mathrm{O}_{2}$ consumption and coronary blood flow to peripheral anti-inflammatory effects and selective precapillary arteriolar vasodilation that favours better end-organ perfusion.

\section{Conclusion:}

Severe haemorrhagic shock causes splanchnic microvascular bimodal response that is dependent on microvascular arteriolar level. First-order A1 and second-order A2 arterioles exhibit progressive vasoconstriction, whereas, third-order A3 and fourth-order A4 arterioles show progressive vasodilation. This bimodal arteriolar microvascular response is associated with erratic blood flow in the microvascular network. Temporary administration of either norepinephrine or vasopressin along with conventional resuscitation exerts deleterious effects at all splanchnic microvascular levels by virtue of their peripheral vascular actions. Cellular cytosolic ATP replenishment maintains a better microvascular profile and therefore a viable option to vasopressors use in progressive haemorrhagic shock.

\section{References}

1. Committee on Trauma (1997) Advanced Trauma Life Support Manual. American College of Surgeons, Chicago.

2. Chaudry IH, Baue, A. (1976) Alterations in adenosine $3^{\prime}$, 5 'monophosphate levels in hemorrhagic shock. Surg Forum 27: 51-3.

3. Van Way CW, Dhar A, Reddy R, Evans L, Wogahn B, et al. (1996) Changes in adenine nucleotides during hemorrhagic shock and reperfusion. J Surg Res 66: 159-166.

4. Meisenberg G, Simmons WH, Eds (1998) in Principles of medical biochemistry. Pp 297-331, Mosby St. Louis.

5. Mazzoni MC, Borgstrom P, Intaglietta M, Arfors KE (1989) Lumenal narrowing and endothelial cell swelling in skeletal muscle capillaries during hemorrhagic shock. Circ Shock 29: 27-39.

6. Fruchterman TM, Spain DA, Wilson MA., Harris PD, Garrison RN (1998) Selective microvascular endothelial cell dysfunction in the small intestine following resuscitated hemorrhagic shock. Shock 10: 417-422.

7. Hurt RT, Matheson PJ, Smith JW, Zakaria ER, Shaheen SP, et al. (2012) Preservation of hepatic blood flow by direct peritoneal resuscitation improves survival and prevents hepatic inflammation following hemorrhagic shock. Am J Physiol Gastrointest Liver Physiol 303: G1144G1152.

8. Vincent JL, De BD (2013) Circulatory shock. N Engl J Med 369: 1726-1734.

9. Beloncle F, Meziani F, Lerolle N, Radermacher P, Asfar P (2013) Does vasopressor therapy have an indication in hemorrhagic shock? Ann Intensive Care 3: 13

10. Wang P, Ba ZF, Chaudry IH (1992) ATP-MgCl2 restores the depressed cardiac output following trauma and severe hemorrhage even in the absence of blood resuscitation. Circ Shock 36: 277-283

11. Hirasawa H, Soeda K, Ohtake Y, Oda S, Kobayashi S, et al. (1985) Effects of ATP-MgCl2 and ATP-Na2 administration on renal function and cellular metabolism following renal ischemia. Circ Shock 16: 337-346.

12. Wang P, Zhou M, Rana MW, Singh G, Ba ZF, et al. (1992) ATP-MgCl2 restores renal microcirculation following trauma and severe hemorrhage. Can J Physiol Pharmacol 70: 349-357.
13. Abdel-Zaher AO, Abdel-Aal RA, Aly SA, Khalifa MM (1996) Adenosine for reversal of hemorrhagic shock in rabbits. Jpn. J Pharmacol. 72: 247-254.

14. Puisieux F, Fattal E, Lahiani M, Auger J, Jouannet P, et al. (1994) Liposomes, an interesting tool to deliver a bioenergetic substrate (ATP). in vitro and in vivo studies. J Drug Target 2: 443-448.

15. Zakaria ER, Ehringer WD, Tsakadze N, Li N, Garrison RN (2005) Direct energy delivery improves tissue perfusion after resuscitated shock. Surgery 138: 195-203.

16. Bohlen HG, Gore RW (1976) Preparation of rat intestinal muscle and mucosa for quantitative microcirculatory studies. Microvasc Res 11: $103-110$.

17. Lee HB, Blaufox MD (1985) Blood volume in the rat. J Nucl Med 26: $72-76$.

18. Olaussen A, Peterson EL, Mitra B, O'Reilly G, Jennings PA, et al. (2015) Massive transfusion prediction with inclusion of the pre-hospital Shock Index. Injury 46: 822-826.

19. Ehringer WD, Niu W, Chiang B, Wang OL, Gordon L, et al. (2000) Membrane permeability of fructose-1,6-diphosphate in lipid vesicles and endothelial cells. Mol Cell Biochem 210: 35-45.

20. Ehringer WD, Chiang B, Chien S (2001) The uptake and metabolism of fructose-1,6-diphosphate in rat cardiomyocytes. Mol Cell Biochem 221: 33-40.

21. Zakaria ER, Spain DA, Harris PD, Garrison RN (2002) Resuscitation regimens for hemorrhagic shock must contain blood. Shock 18: 567-573.

22. Zakaria ER, Hurt RT, Matheson PJ, Garrison RN (2003) A novel method of peritoneal resuscitation improves organ perfusion after hemorrhagic shock. Am J Surg 186: 443-448.

23. Zakaria ER, Garrison RN, Kawabe T, Harris PD (2004) Role of neutrophils on shock/resuscitation-mediated intestinal arteriolar derangements. Shock 21: 248-253.

24. Zakaria ER, Spain DA, Harris PD, Garrison RN (2002) Resuscitation regimens for hemorrhagic shock must contain blood. Shock 18: 567-573.

25. Abou-Khalil B, Scalea TM, Trooskin SZ, Henry SM, Hitchcock R (1994) Hemodynamic responses to shock in young trauma patients: need for invasive monitoring. Crit Care Med 22: 633-639.

26. Scalea TM, Maltz S, Yelon J, Trooskin SZ, Duncan AO, et al. (1994) Resuscitation of multiple trauma and head injury: role of crystalloid fluids and inotropes. Crit Care Med 22: 1610-1615.

27. Abramson D, Scalea TM, Hitchcock R, Trooskin SZ, Henry SM, et al. (1993) Lactate clearance and survival following injury. J Trauma 35: 584-588.

28. Broder G, Weil MH (1964) Excess lactate: An index of reversibility of shock in human patients. Science 143: 1457-1459.

29. Ahlquist RP (1980) Historical perspective Classification of adrenoreceptors. J Auton Pharmacol 1: 101-106.

30. Holmes CL, Landry DW, Granton JT (2003) Science review: Vasopressin and the cardiovascular system part 1 receptor physiology. Crit Care 7: 427-434.

31. Qin K, Sethi PR, Lambert NA (2008) Abundance and stability of complexes containing inactive $G$ protein-coupled receptors and $G$ proteins. FASEB J 22: 2920-2927.

32. Kamiike W, Watanabe F, Hashimoto T, Tagawa K, Ikeda Y, et al. (1982) Changes in cellular levels of ATP and its catabolites in ischemic rat liver. J Biochem. 91: 1349-1356. 